\title{
CORRELAÇÃO DE FATORES ANATOMOPATOLÓGICOS COM A SOBREVIDA DE PACIENTES OPERADOS POR ADENOCARCINOMA COLORRETAL
}

\author{
CORRELATION OF PATHOLOGICAL FACTORS WITH SURVIVAL OF PATIENTS \\ SUBMITTED TO COLORETAL RESECTIONS DUE TO ADENOCARCINOMA
}

\author{
Gilmar Ferreira do Espírito Santo, TCBC-MT'; \\ José Eduardo de Aguilar-Nascimento, TCBC-MT²; \\ Marina Okuyama Kishima ${ }^{3}$; Arlei Takiuchi ${ }^{4}$
}

\begin{abstract}
RESUMO: Objetivo: Avaliar a influência de fatores anatomopatológicos como prognóstico na sobrevida de pacientes operados de adenocarcinoma colorretal. Métodos: Estudo tipo coorte histórica aberta, baseado na análise de 119 pacientes operados de adenocarcinoma colorretal com intenção curativa no Hospital Universitário Júlio Muller, no período de 1984 a 2002. Os dados foram obtidos dos prontuários médicos e de exames anatomopatológicos revisados, sendo submetidos à análise estatística de sobrevida em cinco anos pelo método de Kaplan \& Méier. O reto foi o segmento mais acometido em 44,5\% dos casos. O aspecto macroscópico tumoral predominante foi o ulcerado ou infiltrante (50,4\%), com tamanhos entre 2 e $17 \mathrm{~cm}$, sendo que a maioria dos tumores $(64,7 \%)$ infiltrava até a camada serosa. O número médio de linfonodos analisados foi de $11,8( \pm 7,3)$ por peça cirúrgica, indentificando-se nestes, $42,8 \%$ de metástases. A maioria dos tumores $(85,4 \%)$ era bem ou moderadamente diferenciada. Foram observadas embolização angiolinfática e perineural em respectivamente $51,2 \%$ e $23,5 \%$. Resultados: Não houve significância estatística quanto a morfologia $(\mathrm{p}=0,87)$, tamanho do tumor $(\mathrm{p}=0,56)$ e grau de diferenciação celular $(\mathrm{p}=0,83)$. Os fatores que se correlacionaram com a sobrevida foram o sítio do tumor primário $(\mathrm{p}=0,04)$, a invasão angiolinfática intra-tumoral $(\mathrm{p}=0,02)$, invasão perineural $(\mathrm{p}<0,01)$, a infiltração das camadas $(\mathrm{p}=0,02)$, e o comprometimento linfonodal $(\mathrm{p}<0,01)$. Conclusão: A análise dos fatores anatomopatológicos mostrou correlação significativa da sobrevida com o sítio primário, a camada acometida, invasão perineural, invasão angiolinfática e comprometimento dos linfonodos (Rev. Col. Bras. Cir. 2008; 35(3): 182-187).
\end{abstract}

Descritores: Neoplasias colorretais; Adenocarcinoma; Prognóstico; Taxa de sobrevida.

\section{INTRODUÇÃO}

O adenocarcinoma é o tipo histológico mais freqüente do câncer colorretal, correspondendo a aproximadamente $98,6 \%$. O restante dos tumores primários é representada pelos sarcomas, linfomas, e tumores carcinóides ${ }^{1}$. A ressecção com intenção curativa é possível em aproximadamente $75 \%$ dos pacientes, porém, ainda assim, 27,5\% a $43 \%$ destes desenvolverão recidivas ${ }^{2-3}$. O exame anatomopatológico é de extrema importância na determinação de fatores prognósticos que podem selecionar grupos de pacientes com maior risco de recidiva, candidatos a tratamentos complementares ${ }^{4}$.

A localização do tumor como fator prognóstico foi primeiramente relatada em 1967 por Spratt \& Spjut ${ }^{5}$, que descreveram pior prognóstico quando o tumor era localizado no reto. Desde então os achados são controversos, alguns identificando a localização no reto como pior $^{6-7}$ e outros sem encontrar qualquer significância estatística ${ }^{8}$. Outros achados macroscópicos do exame anatomopatológico que podem ser associados a sobrevida são o tamanho e a morfologia vegetante ou ulcerada do tumor, porem estudos apresentam diferentes resultados ${ }^{9-10}$. A análise da profundidade do acometimento da parede intestinal tem mostrado associação com a recorrência local e à distância, metástases linfonodais e também com menor sobrevida ${ }^{11}$. Os tumores com alto grau histológico (pouco diferenciados e indiferenciados) apresentam correlação independente com pior prognóstico em alguns estudos $^{12-13}$, porém em outros, não há relação de significância estatística com a sobrevida ${ }^{14}$. O subtipo histológico colóide ou mucinoso representa aproximadamente $17 \%$ dos adenocarcinomas e é mais freqüente em pacientes jovens, relacionando-se com maior taxa de recidiva local e pior prognóstico em algumas séries ${ }^{15-16}$. Já outros autores, não encontraram significância prognóstica em pacientes com o mesmo estadiamento tumoral ${ }^{17}$.

A infiltração dos vasos venosos, linfáticos e do espaço perineural, particularmente quando na localização extramural (tecidos peri-cólicos ou peri-retais) é relacionada com a maior ocorrência de metástases linfonodais e hepáticas, aumento na recidiva local e prognóstico desfavorável em várias séries ${ }^{18-20}$.

\footnotetext{
1. Mestre em Saúde e Ambiente pela UFMT; Professor Assistente da Universidade de Cuiabá - UNIC; Cirurgião Oncológico do Departamento de Clínica Cirúrgica da UFMT.

2. Professor Titular; Doutor do Departamento de Clínica Cirúrgica da Faculdade de Ciências Médicas da Universidade Federal de Mato Grosso - UFMT.

3. Professora do Departamento de Anatomia Patológica da Universidade Estadual de Londrina - UEL.

4. Patologista do Instituto de Anatomia Patológica e Citologia de Cuiabá - IAPCC.

Recebido em 10/01/2008

Aceito para publicação em 12/03/2008
}

Conflito de interesses: nenhum

Fonte de financiamento: nenhuma

Trabalho realizado no Departamento de Clínica Cirúrgica da Faculdade de Ciências Médicas da Universidade Federal de Mato Grosso - UFMT. Tese de Mestrado defendida no Instituto de Saúde Coletiva da UFMT. 
O acometimento dos linfonodos regionais é reconhecido pela maioria dos autores, como fator negativo de maior importância na sobrevida dos pacientes operados por adenocarcinoma colorretal ${ }^{21-24}$. O tamanho dos linfonodos não é bom indicador da presença de metástases, que em 50\% a 78\% das vezes acomete espécimes menores que $5 \mathrm{~mm}^{25}$. A quantidade e a localização dos linfonodos comprometidos são, também, fatores imputados como de prognóstico mais sombrio ${ }^{26}$.

Entretanto, há poucas referencias nacionais com análise de sobrevida sobre o assunto ${ }^{22 ; 27,28}$. Dessa forma, um novo trabalho enfocando a associação entre o exame histopatológico e a sobrevida em câncer colorretal com curvas de sobrevida do tipo Kaplan-Meier poderia melhorar as informações nesse campo. Então, o objetivo deste trabalho foi o de avaliar a influência de vários fatores anatomopatológicos como prognóstico na sobrevida de pacientes operados de adenocarcinoma colorretal.

\section{MÉTODO}

O projeto foi aprovado pelo Comitê de Ética do Hospital. Trata-se de estudo tipo coorte histórica aberta, realizado a partir da análise de 119 pacientes portadores de adenocarcinoma colorretal operados com intenção curativa no Departamento de Clinica Cirúrgica do Hospital Universitário Júlio Müller da Universidade Federal de Mato Grosso, Cuiabá, MT, Brasil, no período de 1984 a 2002. Os pacientes foram selecionados dos livros de registro do centro cirúrgico, do arquivo do laboratório de anatomia patológica e dos prontuários médicos arquivados no Serviço de Arquivo Médico Estatístico (SAME) do Hospital, sendo então, seguidos até 31 de dezembro de 2007.

Dos 119 pacientes estudados, 51,8 (48,7\%) eram do sexo masculino e 61 (51,3\%) do feminino. Aidade variou de 14 a 95 anos, com média de 53,6 ( $\pm 15,9)$ anos, e mediana de 54 anos. Todos os pacientes foram previamente estadiados com colonoscopia e/ou enema opaco, RX de tórax e ultrassonografia ou tomografia computadorizada do abdome. Foram consideradas como operação com intenção curativa aquelas em que o cirurgião, do seu ponto de vista, ressecava todo o tumor macroscopicamente visível, mesmo na presença de metástase e desde que esta fosse totalmente removida. Como cirurgia paliativa considerou-se aquela sem finalidade de cura, com remoção completa ou incompleta do tumor primário na presença de metástases não-ressecadas ou, a não remoção do tumor primário com a realização apenas de derivações internas ou externas do trânsito intestinal ${ }^{29}$.

O reto foi o segmento anatômico mais freqüentemente acometido com 53(44,5\%) casos, seguindo-se o cólon esquerdo com 35(29,4\%), o cólon direito com 23 (19,3\%) e o cólon transverso com oito $(6,7 \%)$ casos. Todos os exames anatomopatológicos foram revisados, porém nem todos os fatores histopatológicos poderam ser aplicados uniformemente, devido falta de alguns dados ou inadequação do material arquivado. Em 88 (76,5\%) peças foi observada a morfologia da lesão primária, sendo $28(23,5 \%)$ classificadas como vegetantes e $60(50,4 \%)$ como infiltrativos e/ou estenosantes. O tamanho dos tumores variou de um mínimo de 2,0 cm a um máximo de $17,0 \mathrm{~cm}$, com média de $5,4 \mathrm{~cm}( \pm 2,5)$, em 74 laudos anatomopatológicos com esta descrição. A análise foi feita com a estratificação dos tumores em tamanho até $5 \mathrm{~cm}(\mathrm{~N}=39)$ e maior que $5 \mathrm{~cm}(\mathrm{~N}=35)$. Foram examinados de 3 a 42 linfonodos por exame anatomopatológico, com média de 11,8( $\pm 7,3)$ linfonodos por peça. Em 51(42,8\%) das peças examinadas havia comprometimento linfonodal por metástases, com média de 3,4( $\pm 3,3)$ linfonodos acometidos.

Todos os tumores eram adenocarcinomas e foram subclassificados como usuais ou como mucinosos, quando o componente mucinoso formava $50 \%$ ou mais da neoplasia. O adenocarcinoma usual foi subclassificado como bem (G1), moderadamente $(\mathrm{G} 2)$ ou pouco diferenciado $(\mathrm{G} 3)^{11}$. Para efeito de estudo estatístico e prognóstico, os tumores G1 e G2 foram considerados de baixo e os G3 e mucinosos de alto grau histológico ${ }^{15}$. A maioria dos tumores $(85,4 \%)$ foi classificada como de baixo grau e os 14,6\% restantes como de alto. Em 77 pacientes $(64,7 \%)$ os tumores invadiam a camada serosa e em somente seis casos $(5,0 \%)$, o comprometimento limitou-se à camada submucosa. $\mathrm{Na}$ análise estatística, os tumores com invasão até a camada muscular foram agrupados. Nos 82 tumores em que foi possível análise adequada, a invasão angiolinfática foi encontrada em $42(51,2 \%)$ e a infiltração perineural em $28(23,5 \%)$, todos intra-tumorais.

$\mathrm{O}$ tratamento adjuvante foi efetuado na maioria dos pacientes, sendo a quimioterapia aplicada em 66(68,8\%) e a radioterapia em $32(33 \%)$.

O acompanhamento dos pacientes foi feito através dos prontuários médicos, ambulatório, carta ou contato telefônico. A estimativa de sobrevida foi computada em até cinco anos (60 meses), a contar da data da operação. A análise estatística foi feita com o programa SPSS 15.0, sendo as curvas de sobrevida estimadas pelo método de Kaplan-Meier e a análise comparativa univariada pelo teste de Log-rank. O nível de significância estatística foi de $\mathrm{p}<0,05$.

\section{RESULTADOS}

O seguimento mediano e moda foram de 59 meses (IC 95\% 95,8-117,2) e a sobrevida global em cinco anos foi de $66,82 \%$. A curva de sobrevida pode ser vista na figura 1 .

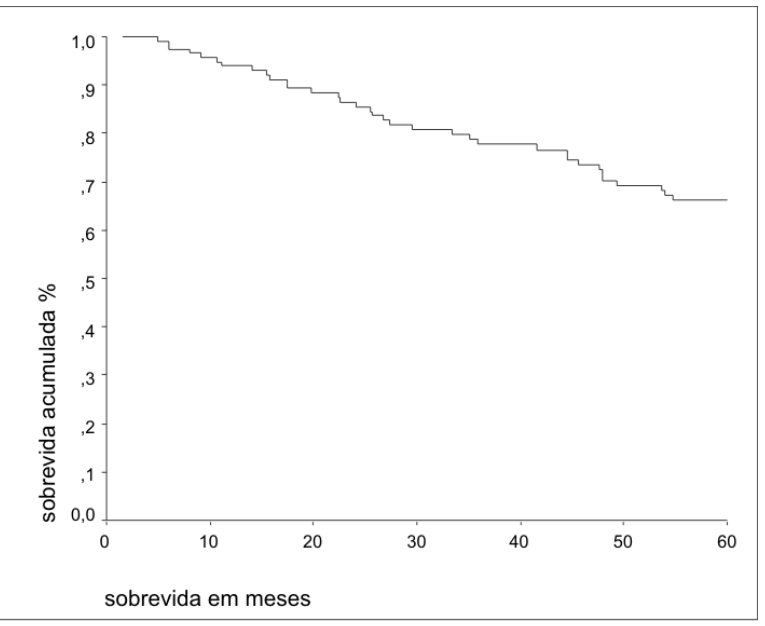

Figura 1 - Curva de sobrevida global não estratificada. 
A sobrevida dos pacientes com tumores localizados no cólon $(75,3 \%)$ foi melhor que a dos com localização no reto $(56,5 \%)$, com significância estatística $(\mathrm{p}=0,04)$, conforme a curva de sobrevida da figura 2. Os fatores macroscópicos, morfologia tumoral $(p=0,87)$ e tamanho do tumor $(p=0,56)$, não apresentaram qualquer influência na sobrevida. A infiltração progressiva das camadas da parede colorretal correlacionouse direta e significativamente com a sobrevida $(p=0,02)$. Enquanto 79,6\% dos pacientes com tumores infiltrativos até a camada muscular sobreviveram cinco anos, aqueles com invasão da serosa tiveram uma sobrevida de 58,7\%. (Figura 3).

Os pacientes com tumores de baixo grau não apresentaram melhor sobrevida que os de alto grau de diferenciação celular $(\mathrm{p}=0,83)$. A invasão angiolinfática intratumoral se correlacionou significativamente, com uma pior sobrevida $(74,2 \%$ x 49,3\%) quando presente $(\mathrm{p}=0,02)$. (Figura 4). Já a invasão perineural, mesmo que intratumoral, representou significante decréscimo na sobrevida $(\mathrm{p}<0,01)$. Quando presente, a probabilidade de sobrevida em cinco anos foi de $40,1 \%$ contra 79,5\%. (Figura 5). O comprometimento dos linfonodos

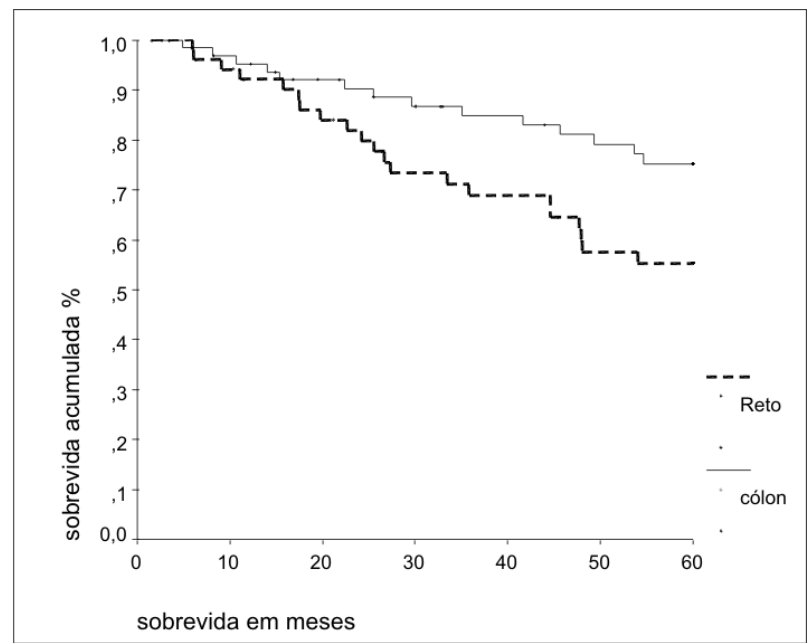

Figura 2 - Curvas de sobrevida segundo o sítio tumoral $(p=0,04)$.

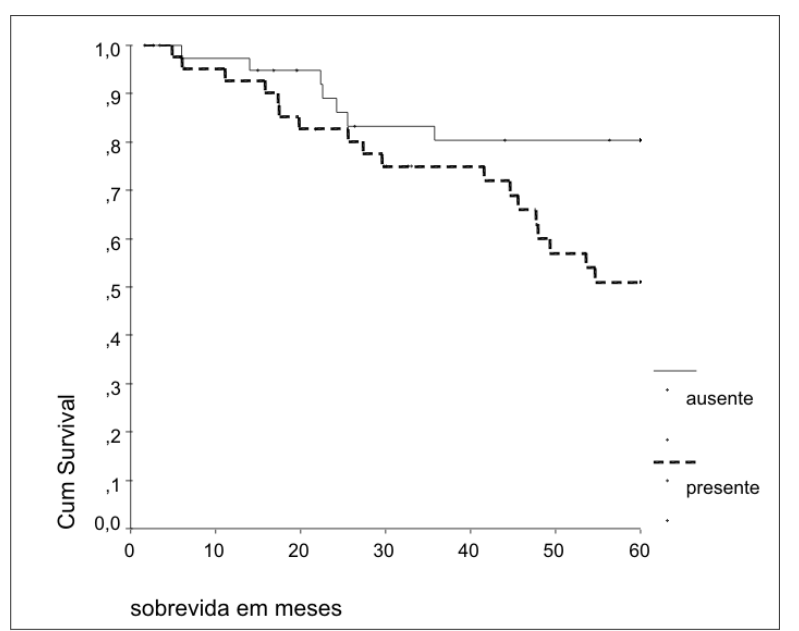

Figura 4 - Curvas de sobrevida quanto à presença de invasão angiolinfática $(p=0,02)$. regionais foi um fator de forte influência na sobrevida $(\mathrm{p}<0,01)$. Os pacientes sem metástases linfonodais tiveram uma estimativa de sobrevida em cinco anos de $81,1 \%$ contra $45,7 \%$ daqueles com linfonodos comprometidos (Figura 6).

\section{DISCUSSÃO}

À luz do grande avanço no campo dos métodos de imagem, genética e marcadores biomoleculares ${ }^{30-33}$, os achados anatomopatológicos ainda são, na maioria das vezes, os mais importantes, acessíveis e fundamentais fatores na determinação da sobrevida e decisão sobre a aplicação de terapia adjuvante. Há porém, grande controvérsia na literatura médica quanto a relação de significância de alguns deles com a sobrevida dos pacientes portadores de câncer colorretal.

O pior prognóstico relacionado aos tumores localizados no reto, principalmente quando abaixo da reflexão peritoneal, ainda permanece controverso. Este pior resultado poderia ser devido à dificuldade técnica em se conseguir limites adequados de ressecção na pelve, como também à biolo-

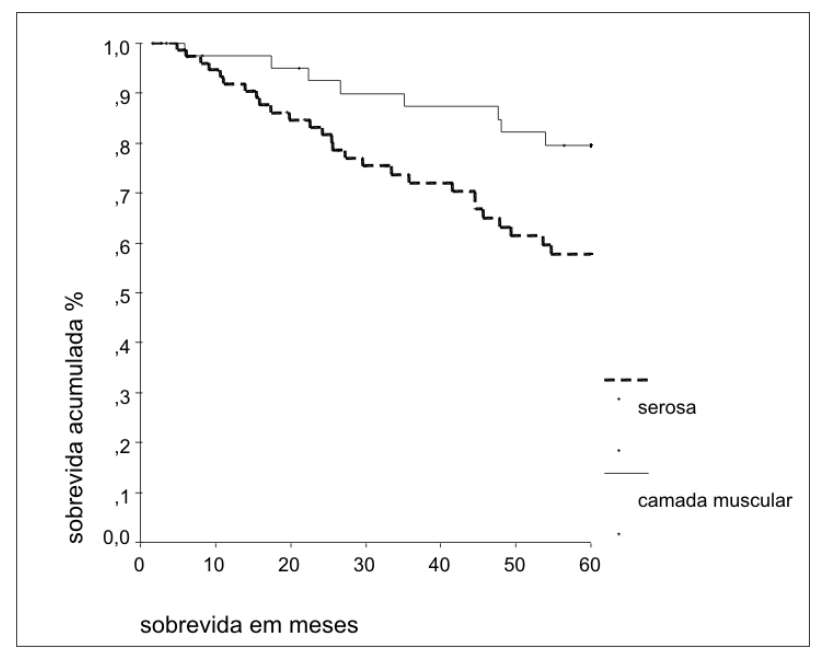

Figura 3 - Curvas de sobrevida conforme invasão até a camada muscular x envolvimento da camada serosa $(p=0,02)$.

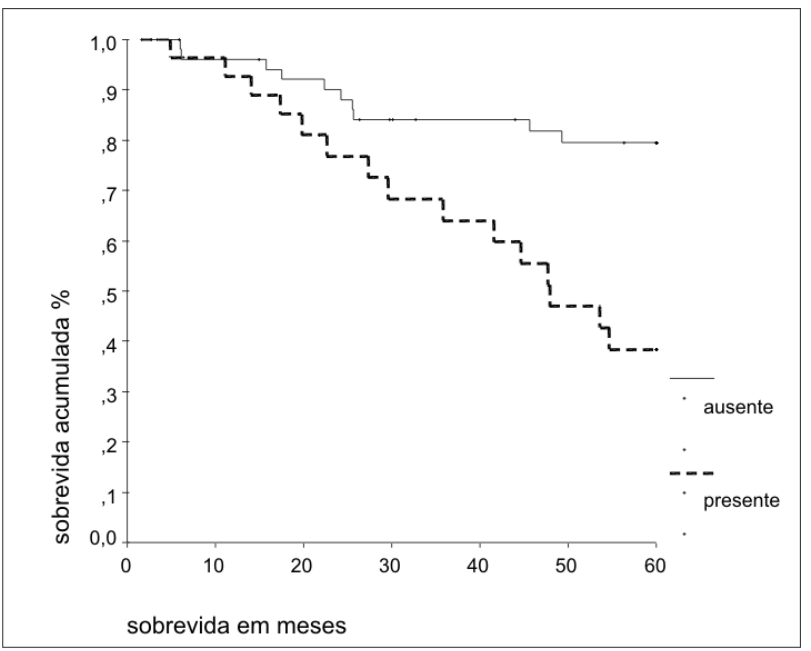

Figura 5 - Curvas de sobrevida na presença de infiltração perineural $(p<0,01)$. 


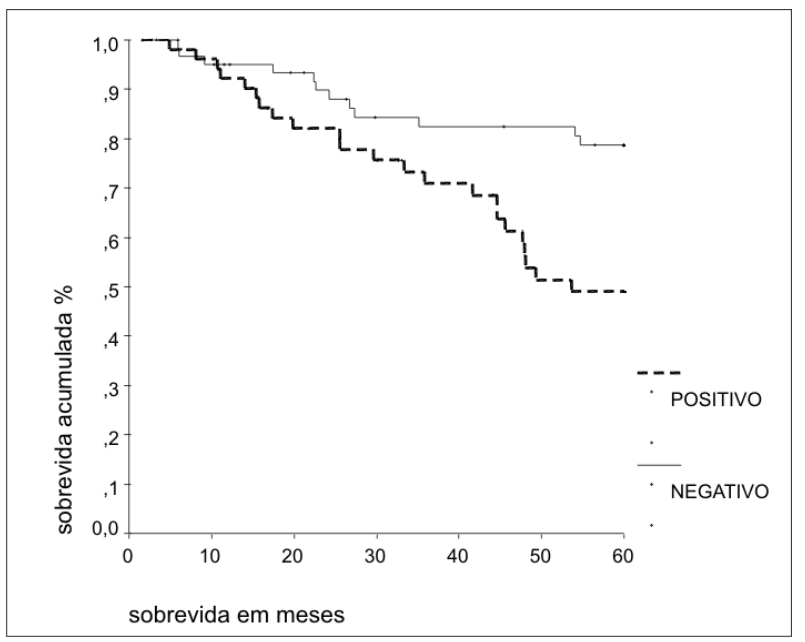

Figura 6 - Curvas de sobrevida conforme o comprometimento linfonodal $(p<0,01)$.

gia tumoral diferente e mais agressiva no câncer retal, o que levaria precocemente a maior índice de recidivas ${ }^{3,34}$. Corroborando com estas justificativas, o sítio retal também teve um pior prognóstico.

A análise macroscópica da morfologia tumoral não significativa como fator prognóstico é corroborada com a maioria dos estudos ${ }^{10,35}$. A invasão da camada serosa reflete o estádio tardio em que é feito o diagnóstico, resultando em uma menor sobrevida ${ }^{11,36}$. Esse aspecto pode ser observado neste presente serie, pois todos os tumores com infiltração máxima limitada à submucosa, sobreviveram cinco anos ou mais.

A importância do grau de diferenciação celular no câncer do reto remonta as primeiras observações feitas por Dukes (1949) ${ }^{37}$. O assunto permanece controverso até hoje, embora, com a utilização da análise multivariada, o grau de diferenciação celular parece não ter significância estatística ${ }^{14}$, 35. As razões para os resultados controversos seriam: a falta de uniformização e a própria interpretação dos critérios de graduação histológica entre os patologistas, a graduação da maioria dos tumores como grau II ou moderadamente diferen- ciados e a variabilidade do grau de diferenciação dentro do mesmo tumor ${ }^{38}$. O grau de diferenciação tem interpretação subjetiva, não fielmente reprodutiva e, conseqüentemente, sua relevância como fator prognóstico pode ser limitada ${ }^{35}$. Nesta casuística, $85,4 \%$ dos tumores analisados foram classificados como de baixo grau, o que certamente influenciou a ausência de significância estatística.

Apesar de não ser avaliada como fator prognóstico na maioria dos estudos, a incidência de invasão dos espaços perineurais nos tumores do reto varia de $14 \%$ a $32 \%$ e correlaciona-se com um maior número de recidivas locais e metástases à distância ${ }^{18-20}$. Um estudo que considerou apenas pacientes com câncer colorretal no estádio "B" de Dukes, identificou através de análise multivariada, a invasão perineural como fator independente de recidiva tumoral e óbito ${ }^{39}$. A incidência de embolização venosa microscópica varia de $17 \%$ a $61 \%$ e a embolização linfática, de $8 \%$ a $73 \%$, aumentando com a progressão do estádio e do grau de diferenciação celular, podendo representar um pior prognóstico ${ }^{20,38,40,41}$. Neste estudo, a análise das curvas de sobrevida demonstra claramente um pior prognóstico dos pacientes com embolização angiolinfática e perineural.

Em 1932, Dukes ${ }^{39}$ já relatava a importância do envolvimento dos linfonodos na sobrevida dos pacientes com câncer do reto. No decorrer desse período, outros estudos confirmaram os resultados daquele autor, como também relacionaram o local e o número de linfonodos com o prognósti$\mathrm{co}^{26}$. Os nossos dados consubstanciam esses relatos. O achado de metástases em linfonodos regionais é sem dúvida, ainda hoje, o principal fator prognóstico relacionado à sobrevida dos pacientes portadores de câncer colorretal submetidos a operação com intenção curativa em concordância com este estudo ${ }^{21,23-24}$.

Dessa forma, pela analise global dos pacientes desta serie, concluímos que o prognóstico de pior sobrevida de pacientes operados de câncer colorretal com intenção curativa, tem nítida associação com a localização no reto, invasão angiolinfática, invasão perineural, infiltração da camada serosa e com comprometimento de linfonodos regionais.

\begin{abstract}
Background: The aim of this study was to evaluate the influence of the pathological aspects as prognostic for survival in patients who underwent resection for colorectal adenocarcinoma. Method: A historical cohort study was conducted based on an analysis of 119 patients with colorectal adenocarcinoma submitted to curative intention resection at the Department of Surgery of the Júlio Müller University Hospital from 1984 to 2002. Data were obtained from medical records. Specimen slides were reviewed and the findings were submitted to the survival analysis using the Kaplan-Meier method. The rectum was the location most frequent found (44,5\%). The predominant macroscopic aspect was ulcerated or infiltrate type (50,4\%), with lengths between 2 and $17 \mathrm{~cm}$, and the majority of the tumors (64,7\%) infiltrated until the serosa layer. The average number of lymph nodes examined in the surgical specimen was 11,8( $\pm 7,3)$ showing 42,8\% of metastatic envolviment. Most of the tumors $(85,4 \%)$ were categorized as either well or moderately differentiated. Angiolymphatic embolization and perineural invasion were observed in $51,2 \%$ and 23,5\% respectively. Results: There was no statistical significance of survival with morphology $(p=0,87)$, length of the tumor $(p=0,56)$ and degree of cellular differentiation $(p=0,83)$. The factors that correlated with survival were location of the tumor $(p=0,04)$, angiolymphatic embolization $(p=0,02)$, perineural invasion $(p<0,01)$, wall infiltration $(p=0,02)$, and lymph node involvement $(p<0.01)$. Conclusion: Survival of patients operated for colorectal adenocarcinoma showed significant correlation with site of the tumor, extension through gut layers, angiolymphatic embolization, perineural invasion, and lymphonodal involvement.
\end{abstract}

Key words: Colorectal neoplasms; Adenocarcinoma; Prognosis; Survival rate. 


\section{REFERÊNCIAS}

1. Disario JA, Burt RW, Kendrick ML, McWhorter WP. Colorectal cancers of rare histologic types compared with adenocarcinomas. Dis Colon Rectum. 1994; 37(12):1277-80. Erratum in: Dis Colon Rectum. 1995; 38(11):1227.

2. Ohlsson B, Breland U, Ekberg H, Graffner H, Tranberg KG. Follow-up after curative surgery for colorectal carcinoma. Randomized comparison with no follow-up. Dis Colon Rectum. 1995; 38(6):619-26.

3. Obrand DI, Gordon PH. Incidence and patterns of recurrence following curative resection for colorectal carcinoma. Dis Colon Rectum. 1997; 40(1):15-24.

4. Compton CC. Colorectal carcinoma: diagnostic, prognostic, and molecular features. Mod Pathol. 2003; 16(4):376-88.

5. Spratt JS Jr, Spjut HJ. Prevalence and prognosis of individual clinical and pathologic variables associated with colorectal carcinoma. Cancer. 1976; 20(11):1976-85.

6. Obrand DI, Gordon PH. Incidence and patterns of recurrence following curative resection for colorectal carcinoma. Dis Colon Rectum. 1997; 40(1):15-24.

7. Chen HS, Sheen-Chen SM. Obstruction and perforation in colorectal adenocarcinoma: an analysis of prognosis and current trends. Surgery. 2000; 127(4):370-6.

8. Ratto C, Sofo L, Ippoliti M, Merico M, Doglietto GB, Crucitti F. Prognostic factors in colorectal cancer. Literature review for clinical application. Dis Colon Rectum. 1998; 41(8):1033-49.

9. Park YJ, Park KJ, Park JG, Lee KU, Choe KJ, Kim JP. Prognostic factors in 2230 Korean colorectal cancer patients: analysis of consecutively operated cases. World J Surg. 1999; 23(7):721-6.

10. Crucitti F, Sofo L, Doglietto GB, Bellantone R, Ratto C, Bossola M, Crucitti A. Prognostic factors in colorectal cancer: current status and new trends. J Surg Oncol. 1991; Suppl 2:76-82.

11. Compton CC, Fielding LP, Burgart LJ, Conley B, Cooper HS, Hamilton SR, et al. Prognostic factors in colorectal cancer. College of American Pathologists Consensus Statement 1999. Arch Pathol Lab Med. 2000; 124(7):979-94.

12. Alexander D, Jhala N, Chatla C, Steinhauer J, Funkhouser E, Coffey CS, Grizzle WE, Manne U. High-grade tumor differentiation is an indicator of poor prognosis in African Americans with colonic adenocarcinomas. Cancer. 2005; 103(10):2163-70.

13. Compton CC. Pathology report in colon cancer: what is prognostically important? Dig Dis. 1999; 17(2):67-79.

14. Blumberg D, Paty PB, Picon AI, Guillem JG, Klimstra DS, Minsky BD, Quan SH, Coher AM. Stage I rectal cancer: identification of high-risk patients - the influence of blood vessel invasion. J Am Coll Surg. 1998; 186(5):574-80.

15. Minsky BD, Mies C, Rich TA, Recht A, Chaffey JT. Coloid carcinoma of the colon and rectum. Cancer. 1987; 60(12):310312.

16. Lee WS, Chun HK, Lee WY, Yun SH, Cho YB, Yun HR, Park SH, Song SY. Treatment outcomes in patients with signet ring cell carcinoma of the colorectum. Am J Surg. 2007; 194(3):294-8.

17. Halvorsen TB, Seim E. Influence of mucinous components on survival in colorectal adenocarcinomas: a multivariate analysis. J Clin Pathol. 1988; 41(10):1068-72.

18. Adachi Y, Inomata M, Kakisako K, Sato K, Shiraishi N, Kitano S. Histopathologic characteristics of colorectal cancer with liver metastasis. Dis Colon Rectum. 1999; 42(8):1053-6.

19. Chok KS, Law WL. Prognostic factors affecting survival and recurrence of patients with pT 1 and pT2 colorectal cancer. World J Surg. 2007; 31(7):1485-90. Epub 2007 May 18.

20. Fujita S, Nakanisi Y, Taniguchi H, Yamamoto S, Akasu T, Moriya Y, Shimoda T. Cancer invasion to Auerbach's plexus is an important prognostic factor in patients with pT3-pT4 colorectal cancer. Dis Colon Rectum. 2007; 50(11):1860-6. Epub 2007 Sep 27.

21. Vieira RA, Lopes A, Almeida PA, Rossi BM, Nakagawa WT, Ferreira FO, Melo CA. Prognostic factors in locally advanced colon cancer treated by extended resection. Rev Hosp Clin Fac Med Sao Paulo. 2004; 59(6):361-8. Epub 2005 Jan 11.

22. Freitas LLL, Lopes A, Rossi BM, Nakagawa WT, Bachega W Jr. Adenocarcinoma de cólon: fatores prognósticos em 60 pacientes tratados com cirurgia curativa. Rev Col Bras Cir. 1996; 24(1):2932.

23. Berberoglu U. Prognostic significance of total lymph node number in patients with T1-4N0M0 colorectal câncer. Hepatogastroenterology. 2004;51(60):1689-93.

24. Chang GJ, Rodriguez-Bigas MA, Skibber JM, Moyer VA. Lymph node evaluation and survival after curative resection of colon cancer: systematic review. J Natl Cancer Inst. 2007; 99(6):433-41.

25. Herrera L, Villarreal JR. Incidence of metastases from rectal adenocarcinoma in small lymph nodes detected by a clearing technique. Dis Colon Rectum. 1992; 35(8):783-8.

26. Tsai HL, Lu CY, Hsieh JS, Wu DC, Jan CM, Chai CY, Chu KS, Chan HM, Wang JY. The prognostic significance of total lymph node harvest in patients with T2-4N0M0 colorectal cancer. J Gastrointest Surg. 2007; 11(5):660-5.

27. Habr-Gama A, Perez RO, Nadalin W, Nahas SC, Ribeiro U Jr, Silva e Sousa AH Jr, Campos FG, Kiss DR, Gama-Rodrigues J. Long-term results of preoperative chemoradiation for distal rectal cancer correlation between final stage and survival. J Gastrointest Surg. 2005; 9(1):90-9; discussion 99-101.

28. Rossi BM, Nakagawa WT, Toshihiko W, Borges AY, Bechara Jr, Vieira RA, René AC, Lopes A, Kowalski LP. Radioterapia e cirurgia na abordagem do cancer do reto.: revisão da literature, fatores prognósticos e resultados de tratamento. Rev Col Bras Cir 1998; 25 (2):81-91.

29. Gentil FC, Lopes A. Princípios de cirurgia oncológica. In: Swartsmann G, editor. Oncologia clínica. Princípios e práticas. Porto Alegre: Artes Médicas; 1991. p. 84-96.

30. Hu WG, Li JW. Feng B, Beveridge M, Yue F, Lu AG, Ma JJ, Wang ML, Guo Y, Jin XL, Zheng MH. Vascular endothelial growth factors $\mathrm{C}$ and $\mathrm{D}$ represent novel prognostic markers in colorectal carcinoma using quantitative image analysis. Eur Surg Res. 2007; 39(4):229-38. Epub 2007 apr 19.

31. Benatti P, Gafà R, Barana D, Marino M, Scarselli A, Pedroni M, et al. Microsatellite instability and colorectal cancer prognosis. Clin Cancer Res. 2005; 11(23):8332-40. Erratum in: Clin Cancer Res. 2006; 12(12):3868-9.

32. Vieira RP, Ribeiro Júnior U, Alves VA, Souza PMS., Ribeiro AVS, Rawet V, Nonogaki S, Gama-Rodrigues J, Habr-Gama A. Correlaçäo das proteínas p53 e KI-67 com o prognóstico de pacientes com adenocarcinoma de reto distal. Rev Bras ColoProctol. 2000; 20(4):246-54.

33. Tomoda H, Baba H, Saito T, Wada S. DNA index as a significant predictor of recurrence in colorectal cancer. Dis Colon Rectum. 1998; 41(3):286-90.

34. Heald RJ. Rectal cancer: the surgical options. Eur J Cancer. 1995; 31A(7-8):1189-95.

35. Bosman FT. Prognostic value of pathological characteristics of colorectal cancer. Eur J Cancer. 1995; 31A(7-8):1216-21.

36. Cianchi F, Messerini L, Comin CE, Boddi V, Perna F, Perigli G, Cortesini C. Pathologic determinants of survival after resection of T3N0 (Stage IIA) colorectal cancer: proposal for a new prognostic model. Dis Colon Rectum. 2007; 50(9):1332-41.

37. Dukes CE. The surgical pathology of rectal cancer. J Clin Pathol. 1949; May;2(2):95-8. 
39. Mulcahy HE, Toner M, Patchett SE, Daly L, O'Donoghue DP. Identifying stage $\mathrm{B}$ colorectal cancer patients at high risk of tumor recurrence and death. Dis Colon Rectum. 1997; 40(3):32631.

40. Dukes CE. The classification of cancer of the rectum. J Pathol Bacteriol. 1932; 35:323-32.

41. Compton CC. Optimal pathologic staging: defining stage II disease. Clin Cancer Res. 2007; 13(22 pt 2):6862s-70s.
Como citar este artigo:

Espírito Santo GF, Aguilar-Nascimento JE, Kishima MO, Takiuchi A. Correlação de fatores anatomopatológicos com a sobrevida de pacientes operados por adenocarcinoma colorretal. Rev Col Bras Cir. [periódico na Internet] 2008; 35(3). Disponível em URL: http:// www.scielo.br/rcbc

\author{
Endereço para correspondência: \\ José E Aguilar-Nascimento \\ Rua Estevão de Mendonça 81 apto 801 \\ Goiabeiras \\ 78043-300 - Cuiabá - MT \\ aguilar@terra.com.br
}

